\title{
Implantable Device Status
}

National Cancer Institute

\section{Source}

National Cancer Institute. Implantable Device Status. NCI Thesaurus. Code C160939.

The status of the implantable device. 